\title{
Cas de COVID-19 confirmés en laboratoire chez les enfants et les jeunes au Canada, du 15 janvier au 27 avril 2020
}

\author{
Dana Paquette ${ }^{1}$, Christopher Bell ${ }^{1}$, Maxime Roy ${ }^{1}$, Lindsay Whitmore ${ }^{1 *}$, Andrea Currie ${ }^{1}$, \\ Chris Archibald ${ }^{1}$, Diane MacDonald ${ }^{1}$, Jennifer Pennock ${ }^{1}$
}

\section{Résumé}

La compréhension de l'épidémiologie de la COVID-19 chez les enfants et les jeunes au Canada aidera à éclairer les mesures de santé publique dans les milieux où les enfants se rassemblent. En date du 27 avril 2020, les gouvernements des provinces et territoires ont fourni à l'Agence de la santé publique du Canada des renseignements détaillés sur 24079 cas, dont 3,9\% ( $n=938$ ) avaient moins de 20 ans. Le taux de détection pour 100000 habitants était plus faible dans ce groupe d'âge (11,9 pour 100 000), par rapport aux personnes âgées de 20 à 59 ans $(72,4$ pour 100000$)$ et de 60 ans et plus (113,6 pour 100 000). L'âge médian des personnes de moins de 20 ans était de 13 ans, et les cas étaient répartis également entre les hommes et les femmes. Parmi les provinces et territoires comptant plus de 100 cas, $1,6 \%$ à $9,8 \%$ des cas étaient âgés de moins de 20 ans. Les cas de ce groupe d'âge étaient plus susceptibles d'être asymptomatiques, soit 10,7\% comparativement à $2,4 \%$ chez les 20 à 59 ans et à $4,1 \%$ chez les 60 ans et plus. Les enfants et les jeunes ont moins souvent connu des résultats graves, mais $2,2 \%(n=15 / 672)$ des cas dans ce groupe d'âge étaient suffisamment graves pour nécessiter une hospitalisation. Selon les renseignements disponibles sur l'exposition, $11,3 \%(n=59 / 520)$ des cas âgés de moins de 20 ans n'avaient aucun contact connu avec un cas. Les résultats du Canada correspondent à ceux d'autres pays.

Citation proposée : Paquette D, Bell C, Roy M, Whitmore L, Currie A, Archibald C, MacDonald D, Pennock J. Cas de COVID-19 confirmés en laboratoire chez les enfants et les jeunes au Canada, du 15 janvier au 27 avril 2020. Relevé des maladies transmissibles au Canada 2020;46(5):136-9.

https://doi.org/10.14745/ccdr.v46i05a04f

Mots-clés : COVID-19, jeunes, enfants, Canada, surveillance
Cette oeuvre est mise à la disposition selon les termes de la licence internationale Creative Commons Attribution 4.0

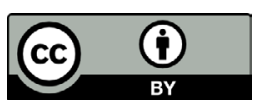

Affiliation

${ }^{1}$ Agence de la santé publique du Canada, Ottawa, ON

\section{${ }^{\star}$ Correspondance : \\ lindsay.whitmore@canada.ca}

Note de l'éditeur : Cet article a été soumis en tant que communication rapide afin de fournir des informations actuelles relatives à la COVID-19 qui pourraient avoir des implications immédiates.

\section{Introduction}

En date du 27 avril 2020, 47327 cas de COVID-19 ont été signalés au Canada. Avec le ralentissement de la croissance des nouveaux cas, les gouvernements provinciaux, territoriaux et fédéral planifient comment et quand alléger certaines mesures de santé publique, notamment le calendrier et les paramètres de réouverture des écoles, des garderies et d'autres lieux où les enfants se rassemblent. Pour éclairer ces décisions, il est important de comprendre l'épidémiologie de la COVID-19 chez les enfants et les jeunes au Canada.

\section{Situation actuelle}

Les données de cette analyse ont été tirées de cas confirmés en laboratoire et signalés à l'Agence de la santé publique du Canada (ASPC) en date du 27 avril 2020. Sur les 47327 cas déclarés à ce jour, des renseignements détaillés ont été fournis à I'ASPC pour 26876 cas. De ce nombre, 24723 ont été confirmés en laboratoire et 24079 comprenaient des renseignements sur l'âge. Parmi ces cas, 3,9\% $(n=938)$ étaient âgés de moins de 20 ans et forment la base de la présente analyse.

Le taux de cas de COVID-19 confirmés en laboratoire était plus faible chez les personnes de moins de 20 ans, à 11,9 pour 100000 (1), comparativement à 72,4 pour 100000 pour les personnes de 20 à 59 ans et à 113,6 pour 100000 pour les personnes de 60 ans et plus $(p<0,001)$. Les taux pour 100000 habitants étaient cohérents $(7,1$ à 11,4 pour 100000$)$ dans les groupes d'âge plus précis pour les enfants de moins de 15 ans, tandis que le groupe des 15 à 19 ans présentait un taux plus élevé (20,7 pour 100 000) (voir le tableau 1). Cette différence peut s'expliquer par le fait que les adolescents sont plus indépendants que les groupes d'âge plus jeunes et donc plus à même de rechercher des contacts sociaux avec leurs 
pairs (2). Il est également important de noter que les différences observées dans les taux d'un groupe d'âge à l'autre peuvent être attribuables, en partie, aux différences dans les tendances des tests en laboratoire selon l'âge.

Tableau 1 : Répartition par âge des cas de moins de 20 ans $(\mathrm{N}=938)$

\begin{tabular}{|c|c|c|c|}
\hline \multirow{2}{*}{$\begin{array}{l}\text { Groupe d'âge } \\
\text { (années) }\end{array}$} & \multicolumn{2}{|c|}{ Fréquence } & \multirow{2}{*}{$\begin{array}{c}\text { Taux pour } \\
100000\end{array}$} \\
\hline & $\mathbf{n}$ & $\%$ & \\
\hline Moins de 1 an & 42 & 4,5 & 11,4 \\
\hline 1 à 4 ans & 109 & 11,6 & 7,1 \\
\hline 5 à 9 ans & 152 & 16,2 & 7,5 \\
\hline 10 à 14 ans & 215 & 22,9 & 11,2 \\
\hline 15 à 19 ans & 420 & 44,8 & 20,7 \\
\hline Total & 938 & 100 & 11,9 \\
\hline
\end{tabular}

Parmi les cas de moins de 20 ans, 50,7 \% étaient des femmes et l'âge médian était de 13 ans. La proportion de cas de moins de 20 ans déclarés quotidiennement est demeurée relativement constante au fil du temps pour les jours où le nombre de cas dépassait 100 (figure 1), allant de 2,1\% à 6,9\%. Des renseignements sur la catégorie d'exposition étaient disponibles pour $55,4 \%(n=520 / 938)$ des cas de moins de 20 ans pour lesquels I'ASPC a reçu des renseignements plus détaillés. De ce nombre, $20,4 \%$ ont été exposés à l'échelle internationale, $9,6 \%$ étaient des contacts de voyageurs des régions touchées, $58,7 \%$ étaient des contacts de cas infectés au Canada et $11,3 \%$ n'avaient aucun contact connu avec des cas. Les groupes d'âge plus jeunes (de 0 à 4 ans, de 5 à 9 ans et de 10 à 14 ans) affichaient un pourcentage plus faible de cas sans contact connu avec un cas $(8,0 \%, 8,3 \%$ et $9,7 \%$, respectivement) comparativement au groupe des 15 à 19 ans, où $15,1 \%$ des cas n'avaient aucun contact connu.

Figure 1 : Courbe épidémiologique du 15 janvier au 27 avril, par groupe d'âge ( $N=22$ 973)

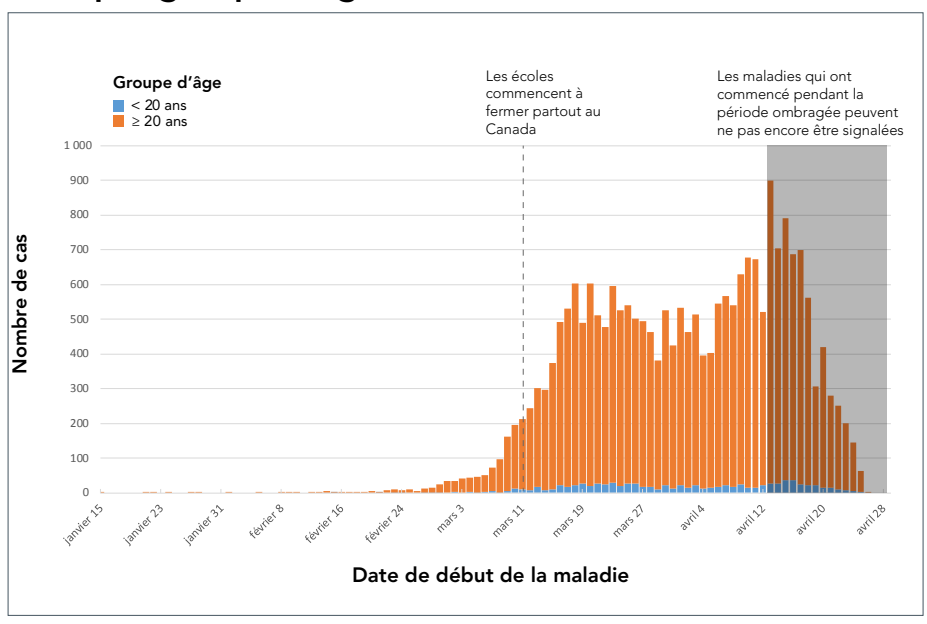

a Si la date du début de la maladie n'était pas connue, on a utilisé la prochaine date disponible pour le prélèvement des échantillons et la date des essais en laboratoire

La répartition des cas dans la catégorie des moins de 20 ans a varié considérablement d'une province et d'un territoire à l'autre $(p<0,001)$ (voir le tableau 2), ce qui peut refléter les différences au niveau de l'épidémiologie locale de la COVID-19 entre les provinces et les territoires et les différences dans les critères d'analyse en laboratoire entre les juridictions. Parmi les juridictions qui ont déclaré plus de 100 cas (à l'exclusion du Yukon, des Territoires du Nord-Ouest, du Nunavut et de l'île-duPrince-Édouard), en Colombie-Britannique, en Saskatchewan, au Manitoba et en Ontario, 5,0 \% ou moins de tous les cas déclarés étaient de moins de 20 ans, tandis qu'en Alberta, au Québec, au Nouveau-Brunswick, en Nouvelle-Écosse et à Terre-Neuveet-Labrador, on a signalé une proportion plus élevée de cas dans ce groupe d'âge (entre $6,8 \%$ et $11,1 \%$ ). Au moment de la rédaction du présent rapport, aucune éclosion n'a été signalée dans les milieux où les enfants se rassemblent (p. ex. garderies, écoles, camps, etc.). Cela peut être dû à la fermeture hâtive des écoles et de certaines garderies.

Tableau 2 : Nombre et proportion de cas de moins de 20 ans, pour les provinces où plus de 100 cas ont été signalés $(\mathrm{N}=937)$

\begin{tabular}{|c|c|c|}
\hline \multirow{2}{*}{ Province $^{a}$} & \multicolumn{2}{|c|}{ Nombre et proportion de cas } \\
\hline & $\mathbf{n}$ & $\%$ \\
\hline Ontario & 328 & 2,2 \\
\hline Alberta & 229 & 8,1 \\
\hline Québec & 226 & 7,3 \\
\hline $\begin{array}{l}\text { Nouvelle- } \\
\text { Écosse }\end{array}$ & 85 & 9,8 \\
\hline $\begin{array}{l}\text { Colombie- } \\
\text { Britannique }\end{array}$ & 25 & 1,6 \\
\hline $\begin{array}{l}\text { Terre-Neuve-et- } \\
\text { Labrador }\end{array}$ & 22 & 8,6 \\
\hline Manitoba & 10 & 4,0 \\
\hline $\begin{array}{l}\text { Nouveau- } \\
\text { Brunswick }\end{array}$ & 8 & 6,8 \\
\hline Saskatchewan & 4 & 3,2 \\
\hline
\end{tabular}

Des renseignements indiquant si les cas étaient symptomatiques ou non étaient disponibles pour 24,7\% ( $n=5$ 939/24 079) des cas pour lesquels I'ASPC a reçu des rapports détaillés. Une plus grande proportion de cas asymptomatiques (10,7 \%) a été constatée dans le groupe des moins de 20 ans, contre $2,4 \%$ dans le groupe des 20 à 59 ans et $4,1 \%$ dans celui des 60 ans et plus. Ces proportions sont probablement des sousestimations des cas asymptomatiques dans tous les groupes d'âge, étant donné que, pendant la période en question, les tests étaient axés sur les populations à haut risque qui étaient symptomatiques.

Des renseignements sur les symptômes étaient disponibles pour $24,6 \%$ ( $n=5$ 912/24 079) des cas pour lesquels l'ASPC a reçu des renseignements détaillés. Parmi ceux qui présentaient des symptômes, les trois symptômes les plus courants différaient selon le groupe d'âge. Les trois symptômes les plus fréquents chez les personnes de moins de 20 ans étaient la toux $(57,0 \%)$, 
l'écoulement nasal $(41,2 \%)$ et les maux de tête (39,4 \%); par rapport à la toux $(74,2 \%)$, les maux de tête $(64,3 \%)$ et la douleur $(56,0 \%)$ chez les personnes âgées de 20 à 59 ans et à la toux $(75,1 \%)$, la faiblesse $(56,2 \%)$ et la fièvre $(51,9 \%)$ chez les personnes âgées de 60 ans et plus. Les autres symptômes courants chez les moins de 20 ans sont la fièvre (36,4\%), le mal de gorge $(34,3 \%)$, la faiblesse $(31,8 \%)$ et les frissons $(30,6 \%)$.

Des résultats graves étaient moins susceptibles de se produire dans le groupe d'âge le plus jeune. L'état d'hospitalisation était disponible pour $57,0 \%$ de tous les cas ( $n=13723 / 24079$ ) pour lesquels I'ASPC a reçu des renseignements détaillés. Parmi les personnes de moins de 20 ans, 2,2 \% ont été hospitalisées, contre $10,4 \%$ chez les 20 à 59 ans et $35,6 \%$ chez les 60 ans et plus. Parmi les personnes hospitalisées de moins de 20 ans, $13,3 \%$ ont été admises à l'unité de soins intensifs. Dans tous les groupes d'âge, ces proportions surestiment probablement la véritable proportion des infections liées à la COVID-19 qui entraînent des résultats graves. En effet, les personnes qui ont présenté des symptômes et des résultats plus graves ont peut-être eu plus de chances d'être testées et de recevoir des résultats que celles qui étaient asymptomatiques ou qui n'avaient que des symptômes légers.

Parmi les personnes de moins de 20 ans, des renseignements sur I'hospitalisation étaient disponibles pour $71,6 \%(n=672 / 938)$ des cas. II n'y a eu que 15 hospitalisations dans ce groupe d'âge, parmi lesquelles les nourrissons de moins d'un an affichaient une plus forte proportion d'hospitalisations que les autres groupes d'âge (voir le tableau 3). Cependant, les chiffres étaient peu élevés dans ce groupe d'âge, et ces résultats devraient être interprétés avec prudence. Dans les 15 admissions à l'hôpital pour les cas de moins de 20 ans, deux cas ont été admis aux soins intensifs, et les deux avaient moins d'un an. Aucun décès n'a été signalé chez les personnes de moins de 20 ans à la date du présent rapport, selon les déclarations publiques des responsables de la santé des provinces et territoires.

\section{Tableau 3 : Statut d'hospitalisation pour les cas de moins de 20 ans $(N=672)$}

\begin{tabular}{|l|r|r|r|r|}
\hline \multirow{2}{*}{$\begin{array}{c}\text { Groupe } \\
\text { d'âge } \\
\text { (années) }\end{array}$} & \multicolumn{2}{|c|}{ Hospitalisation } & \multicolumn{2}{|c|}{ Non hospitalisé } \\
\cline { 2 - 5 } & $\mathbf{n}$ & \multicolumn{1}{|c|}{$\%$} & \multicolumn{1}{|c|}{$\mathbf{n}$} & \multicolumn{1}{c|}{$\%$} \\
\hline $\begin{array}{l}\text { Moins de } \\
1 \text { an }\end{array}$ & 4 & 13,3 & 26 & 86,7 \\
\hline 1 à 4 ans & 0 & 0 & 83 & 100 \\
\hline 5 à 9 ans & 0 & 0 & 118 & 100 \\
\hline 10 à 14 ans & 2 & 1,3 & 153 & 98,7 \\
\hline 15 à 19 ans & 9 & 3,1 & 277 & 96,9 \\
\hline Total & 15 & 2,2 & 657 & 97,8 \\
\hline
\end{tabular}

\section{Conclusion}

Les données présentées dans ce rapport enrichissent notre connaissance de l'épidémiologie de la COVID-19 chez les enfants et les jeunes. Peu de rapports ont été publiés à ce jour, mais selon ce qui est disponible dans la littérature publiée et dans les rapports de surveillance, les résultats canadiens correspondent à ceux d'autres pays. Moins de cas ont été signalés chez les enfants et les jeunes, comparativement aux groupes plus âgés (3-5). Une plus grande proportion d'enfants et de jeunes étaient asymptomatiques et présentaient des symptômes différents de ceux des adultes (6-8). Bien qu'ils soient moins gravement touchés (3-5), il y a quand même eu des hospitalisations, y compris chez les nourrissons $(7,9)$ et, comme aux États-Unis, une plus grande proportion de nourrissons a été hospitalisée comparativement aux enfants et aux jeunes plus âgés (8).

D'après ces constatations et d'autres données, une proportion importante de cas chez les enfants et les jeunes était asymptomatique. Bien que des études préliminaires suggèrent que les enfants sont des sources moins importantes d'infection par le SRAS-CoV-2 que les adultes, une transmission asymptomatique a été constatée (10). II sera donc important de maintenir les principales mesures de santé publique même si certains contrôles sont assouplis. Parmi celles-ci, des comportements comme rester à la maison lorsqu'on est malade, maintenir une distance physique, utiliser des masques non médicaux et se laver fréquemment les mains devraient continuer à être encouragés dans cette tranche d'âge pour prévenir la transmission. De plus, étant donné que des taux plus élevés d'infection confirmée ont été observés chez les 15 à 19 ans, des efforts supplémentaires d'éducation et de renforcement des mesures préventives de santé publique, comme la distanciation physique, pourraient être nécessaires pour cibler ce groupe d'âge plus indépendant et plus mobile.

Pour garantir que la COVID-19 continue à être surveillée chez les enfants et les jeunes, des plans sont en cours pour une surveillance renforcée de la population pédiatrique. Les données seront disponibles à l'aide de plusieurs flux de données en plus des rapports de cas des gouvernements des provinces et territoires. La surveillance accrue fera appel aux bases de données administratives et aux systèmes de surveillance existants, y compris la Base de données sur les congés des patients de l'Institut canadien d'information sur la santé, le Programme canadien de surveillance des infections nosocomiales et le Programme canadien de surveillance pédiatrique. Ces flux de données fourniront des renseignements complémentaires pour surveiller les tendances dans les cas pédiatriques graves, déterminer les facteurs de risque associés à la maladie et évaluer le fardeau de la maladie au sein de cette population.

\section{Déclaration des auteurs}

D. P. - Conceptualisation, ébauche originale, révision et rédaction

C. B. - Conservation des données, analyse officielle, révision et rédaction

M. R. - Conservation des données, analyse officielle

L. W. - Révision et rédaction

A. C. - Révision et rédaction 
C. A. - Révision et rédaction

D. M. - Révision et rédaction

J. P. - Révision et rédaction

\section{Conflit d'intérêts}

Aucun.

\section{Remerciements}

Les auteurs souhaitent remercier les partenaires de surveillance des gouvernements provinciaux et territoriaux, les partenaires nationaux et provinciaux des laboratoires et l'équipe de surveillance du Centre des opérations du portefeuille de la Santé, Agence de la santé publique du Canada.

\section{Financement}

Ce travail a été appuyé par l'Agence de la santé publique du Canada.

\section{Références}

1. Statistique Canada. Profil du recensement, Recensement de 2016. Statistique Canada Catalogue numéro. 98-316X2016001. Ottawa (ON) : Stats Can; date de diffusion le 8 février 2017; mise à jour le 18 juin 2019. https://www12. statcan.gc.ca/census-recensement/2016/dp-pd/prof/index. $\mathrm{cfm}$ ? Lang $=\mathrm{F}$

2. Oosterhoff B, Palmer C. Psychological Correlates of News Monitoring, Social Distancing, Disinfecting, and Hoarding Behaviors Among US Adolescents During the COVID-19 Pandemic. PsyArXiv; March 23, 2020. DOI

3. Public Health England: Weekly Coronavirus Disease 2019 (COVID-19) Surveillance Report. Year: 2020; Week: 17 (Accédé 2020-04-29). https://assets.publishing.service.gov. uk/government/uploads/system/uploads/attachment_data/ file/880925/COVID19_Epidemiological_Summary_w17.pdf
4. World Health Organization (Regional Office for Europe). COVID-19 weekly surveillance report. Data for the week of 13-19 April 2020 (Epi week 16). WHO; 2020 (Accédé 2020-04-29). http://www.euro.who.int/en/ health-topics/health-emergencies/coronavirus-covid-19/ weekly-surveillance-report

5. Australian Government Department of Health. Coronavirus (COVID-19) current situation and case numbers. Canberra (Australia): DOH; 2020 (Accédé 202004-29). https://www.health.gov.au/news/health-alerts/ novel-coronavirus-2019-ncov-health-alert/coronavirus-covid19-current-situation-and-case-numbers

6. Qiu H, Wu J, Hong L, Luo Y, Song Q, Chen D. Clinical and epidemiological features of 36 children with coronavirus disease 2019 (COVID-19) in Zhejiang, China: an observational cohort study. Lancet Infect Dis 2020 Mar;25 (March):S1473-3099(20)30198-5. DOI PubMed

7. Hong H, Wang Y, Chung HT, Chen CJ. Clinical characteristics of novel coronavirus disease 2019 (COVID-19) in newborns, infants and children. Pediatr Neonatol 2020 Apr;61(2):131-2. DOI PubMed

8. Coronavirus Disease 2019 in Children — United States, February 12-April 2, 2020. Morb Mortal Wkly Rep MMWR. 2020;69(14):422-6. DOI

9. Dong $Y$, Mo $X, H u$ Y, Qi X, Jiang F, Jiang Z, Tong $S$. Epidemiology of COVID-19 Among Children in China. Pediatrics 2020 Mar;145(4):e20200702. [Epub ahead of print]. DOI PubMed

10. Hu Z, Song $C, X u$ C, Jin G, Chen $Y, X u X, M a H$, Chen W, Lin Y, Zheng Y, Wang J, Hu Z, Yi Y, Shen H. Clinical characteristics of 24 asymptomatic infections with COVID-19 screened among close contacts in Nanjing, China. Sci China Life Sci 2020 May;63(5):706-11. DOl PubMed 